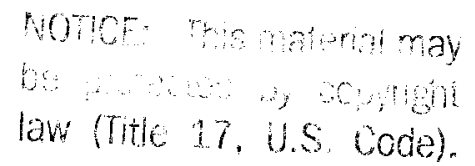

\title{
Coherent polarimeter modules for the QUIET experiment
}

\author{
Kieran A. Cleary, for the QUIET Collaboration \\ California Institute of Technology, Pasadena, CA 91125
}

\begin{abstract}
The Q/U Inaging Experinent (QUIET) is an experimental progran to make very sensitive measurements of the Cosmic Background Radiation (CMB) polarization from the gromul. A key component of this project is the ahility to produce large numbers of detectors in order to achieve the required sensitivity. Using a breakthrough in mm-wave packaging at JPL, a polarimeter-on-a-chip has been developed which lends itself to the mass-production techniques used in the semiconductor industry. We describe the design, implementation and performance of these? polarimeter modules for QUIET Phase 1 and briefly discuss the plans for further module development.
\end{abstract}

Keywords: QUIET, cosmic microwave backgromul, HEMT, colnerent, polarimeter

\section{INTRODUCTION}

The measurement of the polarization of the Cosmic Microwave Background (CMB) is the sulpject of intense interest in the astronomical community. F-node polarization has alrealy been detected 8 . but remains to be fully characterized. The ultimate target for experimentalists, however, is the B-mole polarization due to primordial gravitational waves. Just as the detection of the CMB temperature anisotropies at the $10^{-5}$ level remained a technical challenge for many vears*, the requirement to detect 13 -modes (at levels less than $1 \%$ of the temperature fluctuations) is pushing astronomical instrmuentation towards ever increasing sensitivity.

High electron-mobility transistors (HEMTs) are colerent amplifiers which have been developed for freculenries above $1 \mathrm{GHz}$. They offer advantages of low noise, low power dissipation. high reliability, inherently wide bandwidths, insensitivity to electromagnetic and charged particle radiation, the ability to operate under high signal levels without damage, and operation over a wide temperature range. Because of these qualities. HEMTs have been used for a varicty of gromul-based CMB experiments as well as WMAP and Planck.

The sensitivity of a total power radioneter array is given by

$$
\Delta T=\frac{T_{\mathrm{sys}}}{\sqrt{N \beta T}}
$$

where $T_{s y s}$ is the system noise temperature, $\beta$ is the bandwidth, $\tau$ is the integration time and $N$ is the number of elements in the radiometer array. There are three basic ways to improve the sensitivity: (i) reduce the system noise temperature, $T_{s y s}$ : (ii) increase the bandwidth, $\beta$; or (iii) increase the number of elements in the array, $N$.

Coherent systems such as amplifiers incur a quantum noise penalty which increases with frecfuncy. This sets a. lower limit to $T_{\text {sys }}$ for such systems, which can be written as an equivalent noise temperature, $q=h \nu / h \approx$ $\left[\nu_{\text {Gillz }} / 20\right] \mathrm{K}$, where $h$ is Planck's constant and $h$ is Boltzmann's constant. The state of the art has been $>3 q$ at frequencies in the range $4100 \mathrm{GHz}$ (see, eg. Refs. 9:10). A new ultra-short gate-length IIEMT process ${ }^{1}$ developed by Northrop Grumman Corporation has shown promise of further improvements. An amplifier made using this process has recently demonstrated the lowest room-temperature and cryogenic noise measured to-clate at $W$-band ${ }^{12}$

The performance of individual detectors is thus approaching fundamental linits and while further inprovements are likely, these alone will not provide the sensitivity required for the detection of B-mode polarization. Spectral confusion problems linit the bandwidth of HEMT amplifiers to around $30 \%$. Therefore, the required

\footnotetext{
For further information, e-mail kclearyoastro.caltech.edu

*From the first detection of the CMB dipole to the first all-sky map of the temperature anisotropy took around 20 years.
}

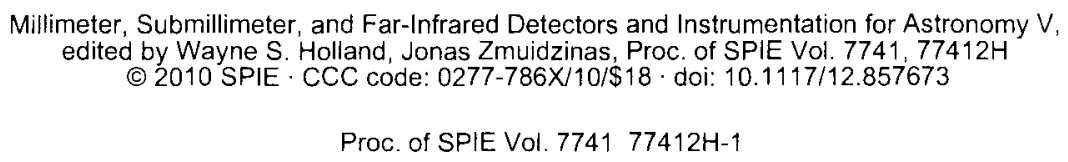

Proc. of SPIE Vol. $7741 \quad 77412 \mathrm{H}-1$ 

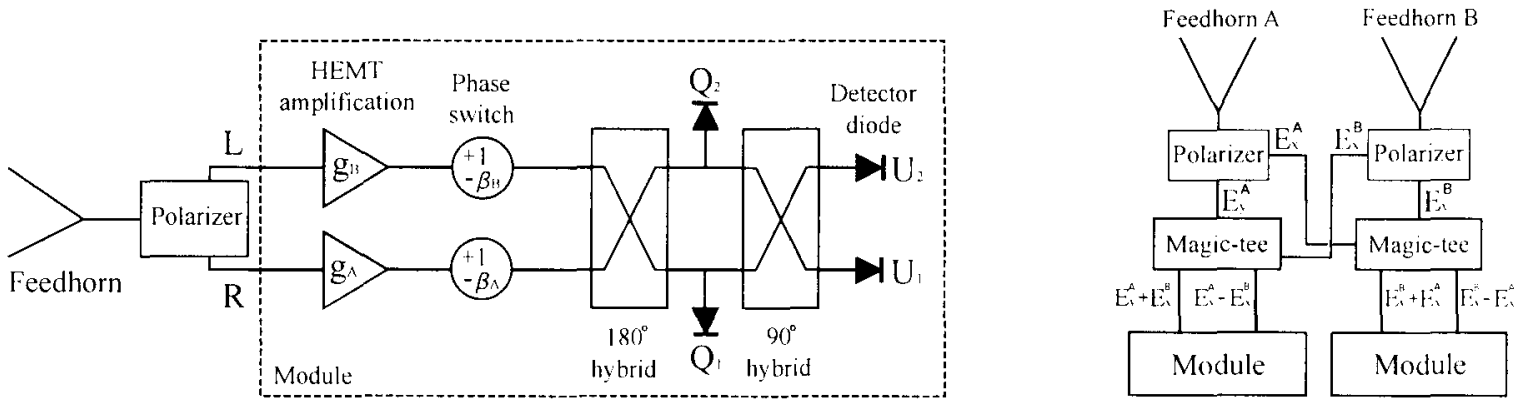

Figure 1. (left) A schematic of the QUIET psoudo-correlation polarimeter. (right) A subset of modules are configured as shown in order to mensum differential total-power.

increase in sensitivity can only be achieved by increasing the number of elements, $N$, in the madiometric array, in tanden with further improvements in the sensitivity of the individual detectors.

The Q/U Imaging Experiment (QUIET) is an experimental program to measure the polarization of the CAlB from the ground using MMIC arrays at two frequencies, deployed at the high and dry Chilean site of Chajnantor in the Atacana desert. QUIET aims to measume or place strong limits on the B-mode polarization due to prinordial gravity waves. In order to acheve the required sensitivity, the experiment exploits a breakthrough in parkaging and miniaturization, which cnables the cost-effective mass-production of MMIC-based polarineter modules, to make large arrays of detectors. Further details about the currently deployed Phase I of (QUDET can be found in Refs. 13-16.

This paper describes the $41 \mathrm{GHz}$ (Q-band) and 95 GHz (W-band) QUIET polarineter modules. The architecture of the pseudo-correlation polarineters is discussed in Sec. 2 while the implementation and packaging is described in Sec. 3. The performance of the modules in Phase I is discussed in Sec. 4 and plans for further module development to enable Phase $1 \mathrm{I}$ of QUIET are briefly reviewed in Sec. 5 .

\section{RADIOMETER ARCHITECTURE}

The expression for radioneter sensitivity given in Echn. (1) above assumes that the receiver gain is constant. In practice, the receiver will experience gain variations which contribute noise, since the detector cannot distinguish between increased power due to changing signal or changing gain. For amplifiers, the power spectrum of these gain variations is of the form $1 / f^{\alpha}$, with a typically in the range 12 . An important concern in the architecture of the polarimeter is the suppression of these $1 / f$ gain fluctuations. Differential receivers can reduce the innpact of amplifier instabilities ${ }^{17}$, however in recent years, the "psendo-correlation" radiometer 18 has been introduced as an improvement over Dicke switching (allows more rapid switching, typically lower loss in hybrid than in ferrite switch and facilitates sideband separation) and used in the WMAP and Planck-LFI instruments.

Figure 1 (left) shows the architecture of the QUIET psendo-comelation polarimeters (the same basic schene is used in both the $44 \mathrm{GHz}$ and $95 \mathrm{GHz}$ modules). A septum polarizer splits the input from a feed horn into left $\left(E_{\mathrm{x}}+i E_{y}\right) / \sqrt{2}$ and right $\left(E_{\mathrm{x}}-i E_{y}\right) / \sqrt{2}$ circularly polarized components, each of which is amplified in a different leg of the poldrineter (where, $E_{\mathrm{x}}$ and $E_{y}$ are orthogonal components of the incident electrical field, with equivalent noise temperatures $T_{\mathrm{x}}$ and $T_{y}$ ). The amplifier gains in each leg are denoted $g_{\mathrm{A}}$ and $g_{\mathrm{B}}$. In one amplifier chain ("leg B"), a synchronous phase switch adds a $180^{\circ}$ phase lag so that when the signals are passed through a hybrid coupler, the outputs give $E_{\mathrm{x}}^{2}-E_{\mathrm{y}}^{2}$, or Stokes $\mathrm{Q}$, after square-law detection and synchronous demodulation. The other amplifier chain ("leg $\Lambda$ ") also inchudes a phase switch (for phase matching) set to a fixed phase state. Stokes I is recovered by taking the average of the diode outputs. Taking the outputs of the first hybrid as inputs to a second hybrid gives $E_{x} E_{y}$, or Stokes U, when the ontputs of the second hybrid are detected and synchronously demodulated. This architecture has the following advantages: (i) both linear polarizations traverse each leg, so that gain fluctuations in the MMIC amplifiers are common-mode and are suppressed by demodulation: (ii) both Stokes $Q$ and $U$ are measured simultaneously, giving an advantage in sensitivity. 
Table 1. Averaged and Demodulated signal for each leg B phase switch state, with leg A phase switched at $4 \mathrm{kHz}$. A factor of $1 / 4$ has been omitted from each expression. The terms involving Stokes $V$ are also omitted for simplicity. The upper (lower) signs correspond to detector diodes Q1 and U1 (Q2 and U2).

\begin{tabular}{|c|c|c|c|}
\hline Phase state & Diodes & Average & Demodulated \\
\hline $\mathrm{PSA} \uparrow$ & $\mathrm{Q} 1(\mathrm{Q} 2)$ & $\frac{1}{2}\left(g_{\mathrm{A}}^{2}+\frac{1+\left(\beta_{\mathrm{B}}^{2}\right.}{2}-g_{13}{ }^{2}\right) I \pm \frac{1-\beta_{\mathrm{B}}}{2} g_{\mathrm{A}} g_{13} Q$ & $\frac{1-{\overline{\beta_{\mathrm{B}}}}^{2}}{4} g_{\mathrm{I}}{ }^{2} I \pm \frac{1+\beta_{\mathrm{B}}}{2} g_{\mathrm{A}} g_{13} Q$ \\
\hline $\mathrm{PSA} \uparrow$ & U1 (U2) & $\frac{1}{2}\left(g_{\Lambda}{ }^{2}+\frac{1+\beta_{\mathrm{B}}^{2}}{2} g_{13}{ }^{2}\right) I \mp \frac{1-\beta_{\mathrm{B}}}{2} g_{\Lambda} g_{\mathrm{B}} U$ & $\frac{1-\beta_{13}^{2}}{4} g_{13}^{2} I \mp \frac{1+, j_{B}}{2} g_{\wedge} g_{\mathrm{B}} U$ \\
\hline PSA $\downarrow$ & Q1 (Q2) & $\frac{1}{2}\left(\beta_{\mathrm{A}}^{2} g_{\mathrm{A}}{ }^{2}+\frac{1+\beta_{\mathrm{B}}^{2}}{2} g_{\mathrm{B}}{ }^{2}\right) I \mp \beta_{\mathrm{A}} \frac{1-\hat{3}_{\mathrm{B}}}{2} g_{\mathrm{A}} g_{13} Q$ & $\frac{1-\beta_{\mathrm{B}}^{2}}{4} g_{\mathrm{B}_{3}^{2}}^{2} I \mp \beta_{\mathrm{A}} \frac{1+\beta_{\mathrm{B}}}{2} g_{\mathrm{A}} g_{\mathrm{B}} Q$ \\
\hline $\mathrm{PSA} \downarrow$ & $\mathrm{U1}$ (U2) & $\frac{1}{2}\left(\beta_{\Lambda}^{2} g_{\mathrm{A}}^{2}+\frac{1+\beta_{\mathrm{B}}^{2}}{2} g_{\mathrm{B}}^{2}\right) I \pm \beta_{\mathrm{A}} \frac{1-\beta_{\mathrm{B}}}{2} g_{\mathrm{A}} g_{\mathrm{B}} U$ & $\frac{1-\beta_{\mathrm{B}}^{2}}{4} g_{\mathrm{B}}^{2} I \pm \beta_{\mathrm{A}} \frac{1+\beta_{\mathrm{B}}}{2} g_{\mathrm{A}} g_{13} U$ \\
\hline
\end{tabular}

In practice, any transmission imbalance (denoted $\beta_{A}$ and $\beta_{13}$ in Fig. 1) between the phase switches can canse $\mathrm{I} \rightarrow \mathrm{Q} / \mathrm{U}$ leakage. This can be seen in the first two rows of Table 1 , which show the demodulated and average ontput at each diode, with the leg $A$ phase switch fixed in one state. However, if $\operatorname{leg} A$ is also phase switched. then the difference of the demodulated detector outputs is free from this systematic error. This operation is referred to as double-demodulation. The second two rows of Table 1 give the diode outputs for the other phase state of the leg $\Lambda$ phase switch. It can be soen that the difference of the denodulated ontputs at a given dioxle between the two leg $\Lambda$ phase states is free from $\mathrm{I} \rightarrow \mathrm{Q} / \mathrm{U}$ leakage.

A subset of modules in the $Q$ - and $W$-band arrays make use of a modified input scheme in order to measure the differential total power between adjacent feed horns. Fig. 1 (right) shows a schematic of these "T"T modules". A polarizer located after foedhorn " $\Lambda$ " ontputs both linear polarizations, $E_{x}^{\Lambda}$ and $E_{y}^{A}$. One of these polarizations, $E_{\mathrm{y}}^{\mathrm{A}}$, enters a magic-tee and is combined with the orthogonal polarization from an arljacent feedhorn, $E_{\mathrm{x}}^{\mathrm{B}}$. The magic-tec outputs are coupled to a module input with the result that, after detection and synchromous denoolulation, the $Q$ diodes of that module measure $E_{\mathrm{y}}^{\Lambda}$ and $E_{\mathrm{x}}^{\mathrm{B}}$, while the $Q$ diodes of the adjacent TT module measme $E_{\mathrm{x}}^{\Lambda}$ and $E_{\mathrm{y}}^{\mathrm{I3}}$. By differencing the ont put of $Q$ diodes from adjacent 'T'T modules, the differential todal power in both polarizations, $E_{\mathrm{x}}^{A}-E_{\mathrm{x}}^{\mathrm{l3}}$ and $E_{\mathrm{y}}^{\mathrm{A}}-E_{\mathrm{y}}^{\mathrm{I3}}$, can be measured.

\section{MODULE DESIGN}

The correlation receivers which have been developed for CMB polarinetry are expensive and time-consuming to make. For example, the CAPMAP polarineter ${ }^{19}$ shown in Fig. 2 (left) costs around $\$ 10 \mathrm{k}$ and requires aromed 50 hours for checking and characterizing. Mannal tuning of each component in order to achieve gain and phase matching is labor-intensive. These polarimeters are therefore not in practice scalable to the mumber of detectors necessary to cletect primordial B-nnode polarization fluctuations. For the QUETT project, a breakthongh in polarimeter packaging and clevolopment was required. Two key techniques were adopted from the semiconductor industry: (i) integration of many functions on a single chip, and (ii) integration of multiple chips in a single module. Automated die attachnent and wire bonding equipment enables the mass production of such modules. The result is a "polarimeter on a chip", containing all the components necessary to amplify a polarization signal with very low noise, taking mu-wave inputs and providing DC outputs.

Figures 3 and 4 show the QUIET W- and Q-band polarimeter module packages. The packages are manufactured from brass and then nickel and gold electro-plated. Feedthough pins supply bias to the active devices and allow the detected voltages to be rear out. Two waveguide input ports are machined into the module lids and accept left- and right-circularly polarized inputs from a septum polarizer. Figures 3 and 4 also show a close-up of the module interiors. The first modules to be built were assembled by hand, however 65 of the $90 \mathrm{~W}$-band modules were assembled using automated die-attachment to epoxy the components in place. Gold ribbon bonds $(2 \times 0.25$ mil cross-section $)$ are used to connect substrates to feed-throughs and devices. Wire bonding was performed using a manual bonding machine, however this will be an antomated process in future phases of the QUIET project. The RF and bias substrates are made from gold on alumina. Unlike at $W$-band, the $Q$-band modules use standard printed circuit board to distribute the device biases and readouts.

Proc. of SPIE Vol. $774177412 \mathrm{H}-3$ 

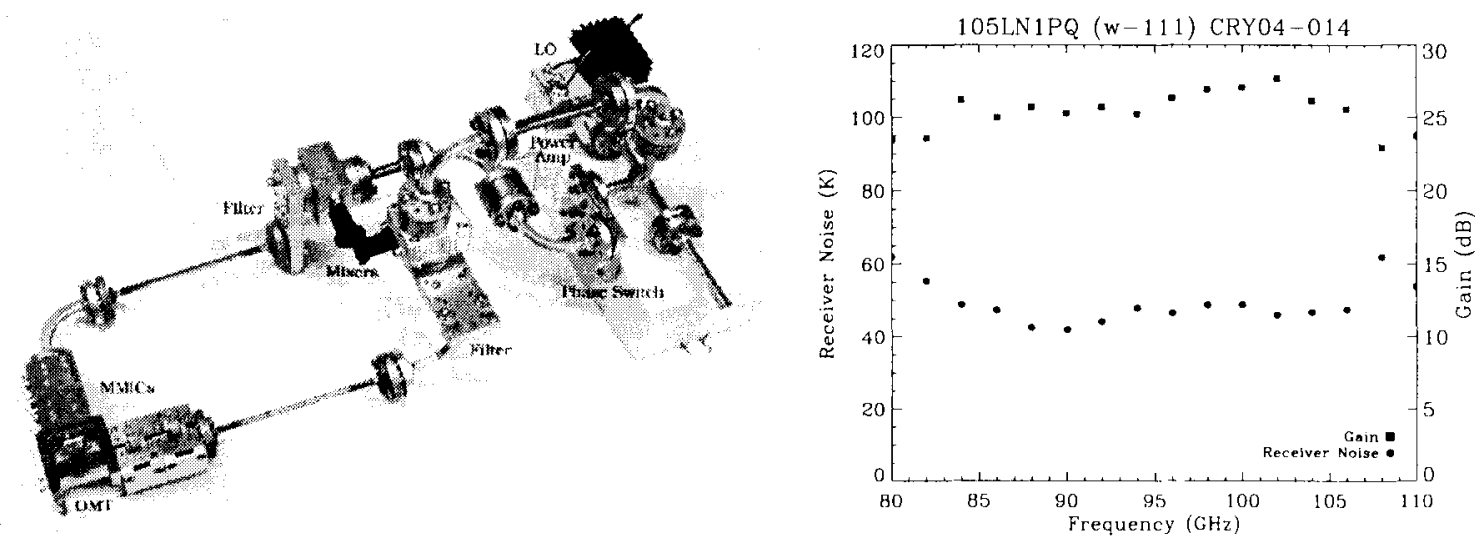

Figure 2. (left) The CAPMAP coherent polarimeter shown here is expensive and time-consuming to characterize. The QUIET polarimeter modules integrate these components into a single package in an antomated assembly process. (right) The performance of a $\mathrm{W}$-band MNIC $1 \mathrm{~N} A$ is shown which exhibits 10 60 $\mathrm{K}$ noise temperature and 25 ab gain when cooled to $25 \mathrm{~K}$.
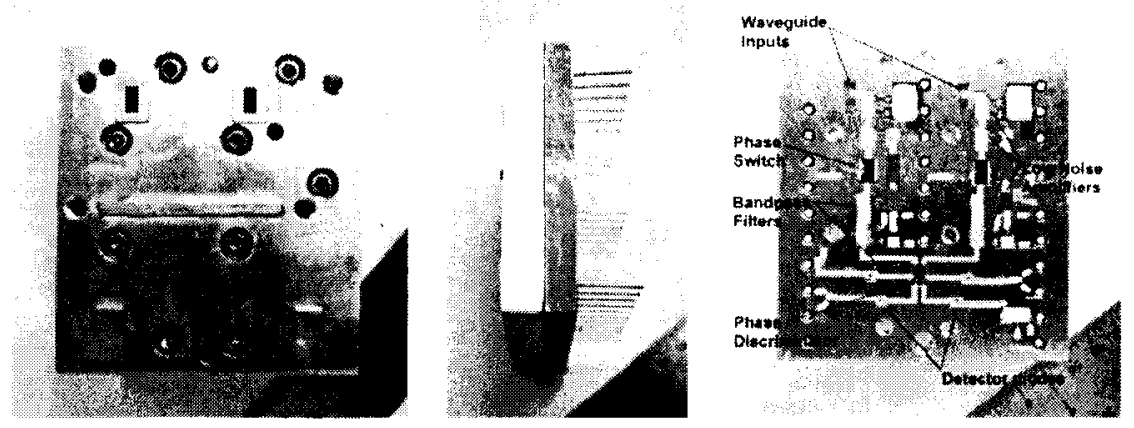

Figure 3. The QUEET W-band polarimeter $3.2 \times 2.9 \mathrm{~cm}$ module is shown from above (left) and from the side (middle). The package is also shown with the lid removed, revealing the module components (right).

Both the Q-and W-band modules have the same basic design. Waveguide input probes convert the incident ficld from the polarizer into time-varying voltages on the 50 ( 2 microstrip transmission lines. On each polarineter leg, these signals are first anplified using a MMIC amplifier chip, selected for its low-noise characteristics. The availability of MMIC low noise anplifiers (LNAs) is key to the performance of the QUlET modules. Indium phosphide (InP) HEMT amplifiers have shown excellent low-noise performance in the QUIET bands, with neasured noise temperatures of $20 \mathrm{~K}$ at $41 \mathrm{GHz}$ and $50 \mathrm{~K}$ at $95 \mathrm{GHz}$ (e.g. seo Fig. 2, right) when cooled to 20 $\mathrm{K}$. This first LNA is followed by a second anplification stage and a phase switch. The phase switches are InP MMIC chips based on PiN ( $p$-type intrinsic, $n$-type) diodes. The diodes act as variable resistors in the circuit. switching to a low-resistance state when forward biased and a high-resistance state when reverse biased. The large impedance difference in these two states is used to block one available signal path in favor of another which is $180^{\circ}$ ont of phase with the first. With both diodes turned on, there is effectively an open circuit in the RF path which is useful for diagnostics. After the phase switch, the signals are filtered to remove ont-of-band gain peaks and then enter the third and final anplification stage. The signals from each leg are combined in a $0^{\circ}$, $90^{\circ}, 180^{\circ}$ hybrid, fabricated on InP, which allows sinultaneous detection of Stokes Q and U. At each of the four hybrid outputs, a band-defining filter is applied and then the signals are detected using commercially-available GaAs Schottky diodes. 

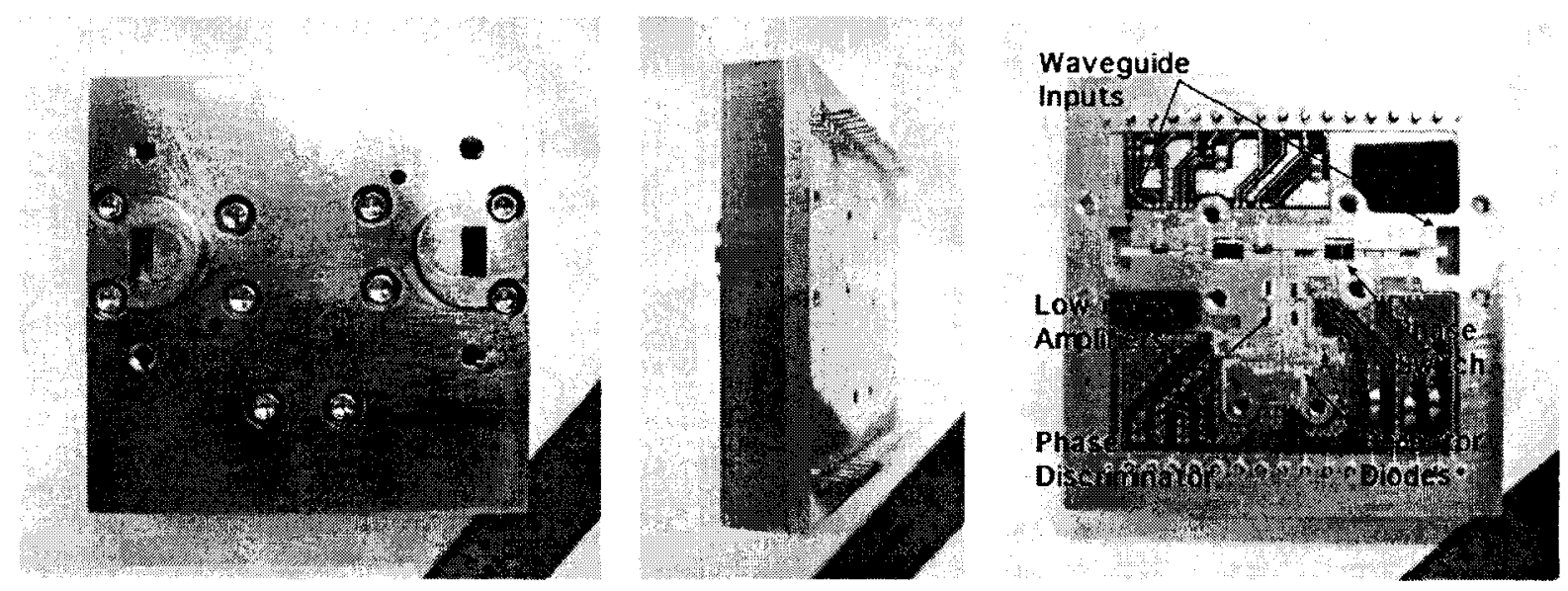

Figure 4. The QUIET Q-band polatimeter $5.1 \times 5.1 \mathrm{~cm}$ module is shown from above (left) and from the side (midde). The package is also shown with the lid removed. revealing the module components (right).

\section{MODULE PERFORMANCE}

The modules bave been extensively characterized in the field and in this section we disenss their performance in terms of sensitivity, $1 / f$ knee frequency and $I \rightarrow Q / U$ leakage.

Ecrn. I gives the sensitivity of a total power radiometer. The $\Delta T_{\text {min }}$ for a psendo-comelation polarimeter medsuring $T_{\mathrm{x}}-T_{\mathrm{y}}$ is $\sqrt{2}$ higher, but with the (now standard) definition $Q=\left(T_{\mathrm{x}}-T_{\mathrm{y}}\right) / 2$, we find the minimm detectable $Q$ is $\Delta Q_{\text {min }}=T_{\text {sys }} / \sqrt{2 \beta \tau}$, and similarly for $\Delta U_{\text {min }}$. Moreover, one can show that the noise in $U$ is nncorrelated with that in $Q$ for an ideal polarineter. Therefore, we define the module sensitivity to polarization as $T_{\text {sys }} /(2 \sqrt{1 i \tau})$. The $W$-band module sensitivity measured on the sky is worse than expected based on the noise temperature of individually packaged input LNAs. Based on the white noise level of data taken on the sky the average module sensitivity is $0.25(0.5) \mathrm{mK} \sqrt{s}$ for $Q$-band (W-band), giving an array sensitivity of $64 \mu \mathrm{K} \sqrt{s}$ $(57 \mu \mathrm{K} \sqrt{s})$.

As discussed in Sec. 2, the QUIET modules use a correlation architecture to suppress the $1 / f$ noise from gan Huctuations in the amplifiers. The anplitude of these gain fluctuations is characterized by a "knee frequency" at which the noise power due to $1 / f$ gain fluctuations equals the white noise level. Further, a double-demodulation scheme is used to reduce the $\mathrm{I} \rightarrow \mathrm{Q} / \mathrm{U}$ leakage due to any mismatch in the transmission of the platse switches. Since double-denodulation reduces the polarized offsets due to $\mathrm{I} \rightarrow \mathrm{Q} / \mathrm{U}$ leakage, this also has the cffect of suppressing the $1 / f$ knee frequency. Fig. 5 shows an example of the power spectrum from a single cletector diode during a $Q$-band constant-elevation scan. The total power is derived from an average of the detector diode output and so has no immunity from gain fluctuations in the receiver. However, the knee-frequencies for the demodulated and donble-rdemodulated cases are significantly reduced compared to that for the total power Fig. 6 shows a histogram of the median $1 / f$ knee-frequency (double-demodulated) of each module resulting from atmospheric and any residual gain fluctuations. The knee-frequencies are typically below the slowest telescope scan frequency of $50 \mathrm{mHz}$ and so the loss of sensitivity due to $1 / f$ noise is minimized.

As can be seen from Fig. 6 (left), the knee frequencies for the $\mathrm{Q}$ diodes are higher than those for the $\mathrm{U}$ dionles, at Q-band. Positive retum loss at low frequencies at the input of the Q-band module (discovered late in the building phase) forced the use of an unoptinized polarizer design which allowed the reflected power to terminate on the sky (rather than set up oscillations) but which had worse leakage performance at the upper end of the band. This leakage causes polarized offsets that increase the $1 / f$ knee frequencies.

The polarizer leakage has two components: one due to an imbalance between the transmission of $E_{\mathrm{x}}$ and $E_{y}$, the other caused by crosstialk between the polarizer outputs. The former affects only $Q$ diodes and the latter affects both $Q$ and $U$ diodes. In praction, the leakage is dominated by the transmission imbalance and 


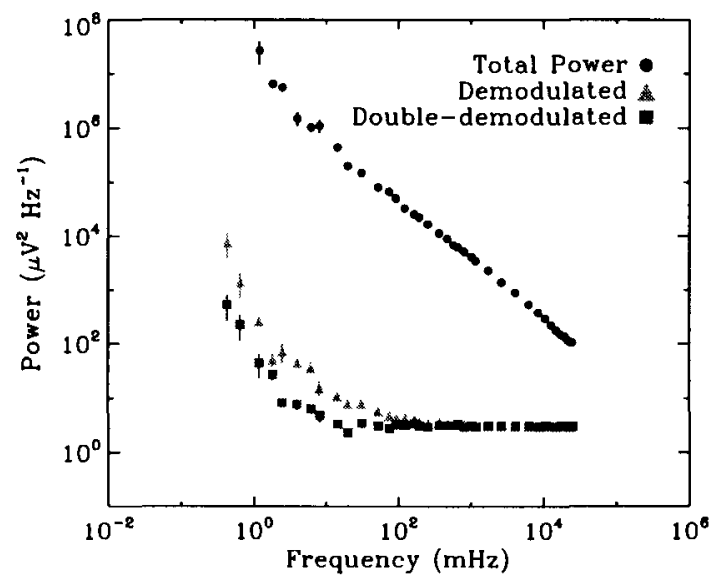

Figure 5. Demodulation and double-demodulation of the diode outputs suppresses the $1 / f$ knee-frequency. conpared to that for the total power.
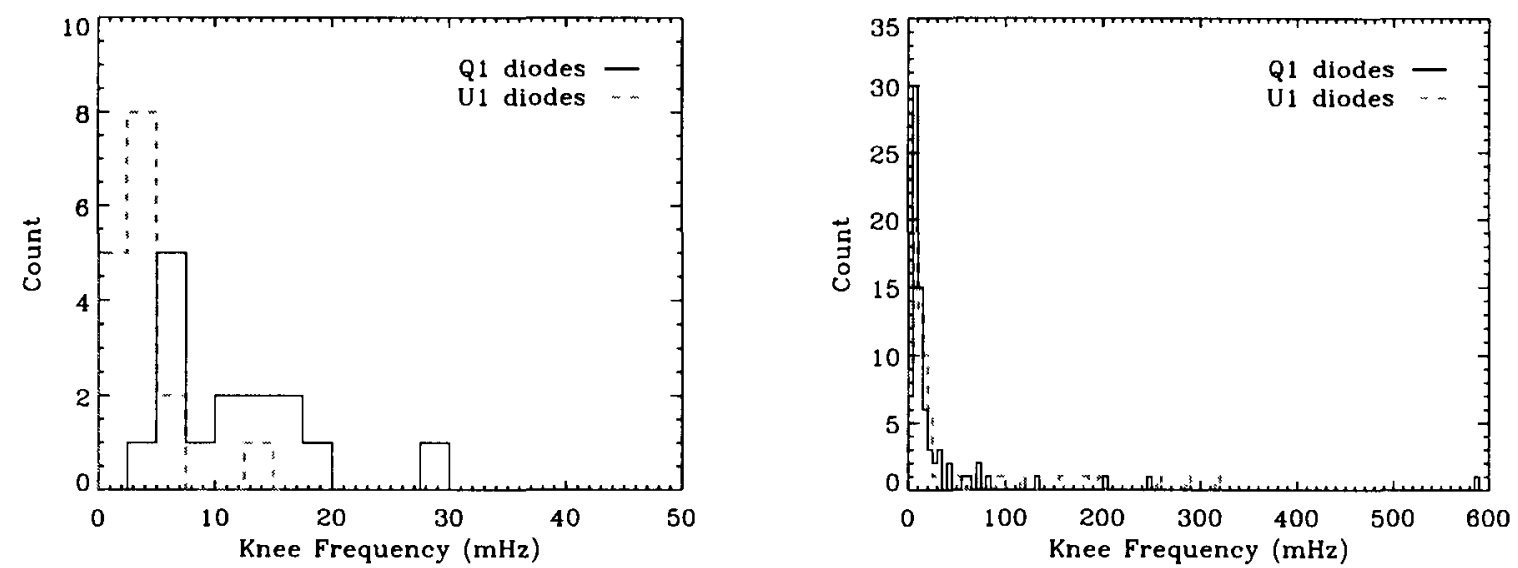

Figure 6. The median $1 / \int$ knee-frequencies are shown for Q-band (left) and W-band (right). As described in the text, the knee frequencies are higher for $\mathrm{Q}$ diodes than $\mathrm{U}$ diodes at $\mathrm{Q}$-band due to $\mathrm{I} \rightarrow \mathrm{Q} / \mathrm{C}$ leakage. However, the knee frequencies are still typically slower than the slowest telescope scan frequency of $50 \mathrm{mH} z$. 


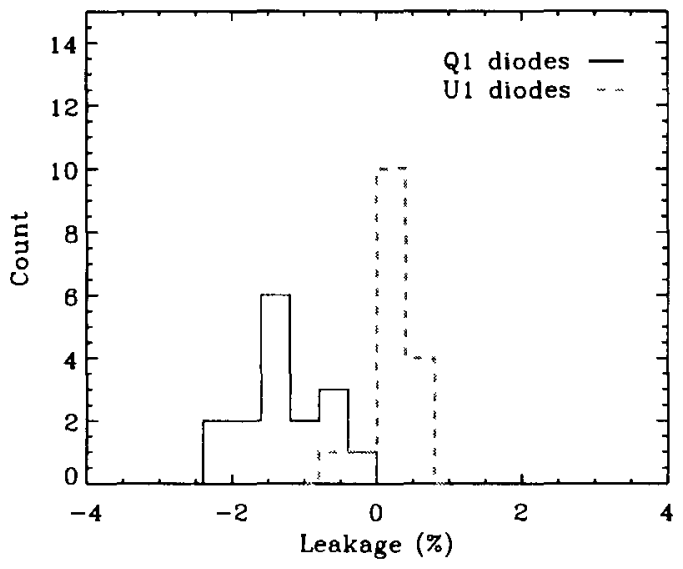

Figure 7. The $I \rightarrow Q / U$ leakage values are shown for $Q$ and $U$ diodes, at, Q-band.

so is seen primarily in the $Q$ diodes. This is why the Q-diodes exhibit higher $1 / f$ knee frequencies than the $U$ diodes at Q-band. Fig. 7 shows the leakage values for Q-band, measured using elevation scans of the atmosphere ("sky-rlips")

The transmission inbalance which gives rise to leakage in the Qband polarizer peaks at high frequencies within the polarizer pass band. Measumements marle using sources with rising spectra (flux density, $S \times v^{\prime r}$, $n>0)$ exhibit greater leakage than those with flatter spectra. The leakage values shown in Fig. 7 were obtained from clevation scans of the atmosphere which, in the passhand of the Q-band modules, is dominated by the tail of the $\mathrm{O}_{2}$ line. Since the black-body CMB will exhibit a flatter spectrum than this, the leakage values we adopt. from the sky-dip measurements are therefore conservative

The W-band leakages are of smaller magnitude and have similar distributions for $Q$ and $U$ diodes. A predininary estimate of the W-band diode leakage distribution indicates typical values of $<1 \%$.

\section{FUTURE PLANS}

In Phase II of QUIET, the sensitivity of the experiment will be improved lyy increasing both the number of modules and the sensitivity of the individual modules. The QUIET Phase I Q- and W-band modules were built using a mixture of automated and manual assembly techniques. The InP MMICs and alumina substrates were cpoxied to the module housing using antomated die-attachnent equipment; however, wire bonding was perforned manually. In order to enable the fully automated assembly which will be required to produce hundreds of modules. autonated wire bonding equipunent will be used.

The sensitivity of the individual modules can be inproved in two ways: by increasing the bandwidth and by reducing the noise temperature of the input. LNAs. The QUIET design bandwidth is 15 GHz. however the combination of LNA and hybrid compler used in the Phase I design limited the bandwidth to at most 12 GHz. For Phase II, alternative designs of hybrid compler and LNA will be explored in order to increase the bandwidth.

The W-band module sensitivity measured on the sky is worse than expected based on the noise temperature of individually packaged input LNAs. The cause of this degradation will be investigated for the next generation of modules. A new 35 nm gate-length fabrication process ${ }^{11}$ at Northrop Grumman Corp. has resulted in inprovements in the noise of HEMT transistors at $Q$ - and W-band. JPL has designed MMllCs nsing these new devices and are in the process of characterizing them at room and cryogenic temperatures. A series of prograns is underway at Caltech and JPL. funded by the Keck Institute for Space Studies (KISS) and NASA, aiming to optimize the performance of these new devices for cryogenic operation. The improved LNAs resulting from these activities will be used in the next generation of QUIET polarimeter modules. 


\section{ACKNOWLEDGMENTS}

The seven U.S. QUIET Institutions are funded through an NSF Award (AST-0506648). Support also provided by AST-04-49809, DE-AC02-05CH11231, JSPS KAKENHI (A) 20244041, NSF PHY-0355328 and by the Strategic Alliance for the Implementation of New Tech- nologies (SAINT). Some work presented was performed on the Joint Fermilab - KICP Supercomputing Cluster, supported by grants from Fermilab, Kavli Institute for Cosmological Physics, and the University of Chicago. We are particularly grateful to Mary Soria (JPL), April Camphell (Wedgeway) and Heather Owen (JPL) for the assembly of the QUIET modules, and to the engineers who maintained and operated the telescope: J. Cortes, C. Jara, F. Munoz, and C. Verdugo.

\section{References}

[1] Kovac, J. M., Leitch, E. M. , Pryke. C. . Carlstrom, J. F. , Halverson, N. W. , Holzapfel, W. L.. "IDetection of polarization in the cosmic microwave background using DASI", Nature, 420, $772-787(2002)$

[2] Readhead, A. C. S., Myers, S. T., Pearson, T. J. Sievers, J. L. , Mason, B. S. , Contaldi, C. R. , Bond, J. R., Bustos, R. , Altanirano, P., Achermann, C. Bronfman, L. , Carlstrom, J. E. , Cantwright, J. K. . Casassus, S. Dickinson. C. Holzapfel, W. L. Kovac, J. M. Le itch, E. M. May. J. Padin, S. , Pogosyan. D. , Pospieszalski, M. , Pryke, C. , Reeves, R. Shepherd, M. C. , Torres, S., "Polarization Observations with the: Cosmic Backgromed Imager", Scienee, 306, 836-814 (2004)

[3] Leitch, E. M. Kovac, J. M. , Ialverson, N. W., Carlstrom, J. E., Pryke, C., Smith, M. W. E., "Degree Angular Scale Interferometer 3 Year Cosmic Microwave Background Polarization Results", Ap. 624. 10-20 $(2005)$

[1] Montroy, T. E., Ade, P. A. R., Bock, J. J. Bond, J. R. , Borrill, J., Boscaleri, A., Cabella. P. . Contaldi. C. R. Crill, B. P. , de Bernardis, P. , De Gasperis, G., de Oliveira-Costa, A., De Troia, G. . di Stefano, G. Hivon, E. Jaffe, A. II. Kisner, T. S., Jones, W. C., Isunge, A. E., Masi, S., Mauskopf, P. I). , Mac'Tavish, C. I., Melchorri, A., Natoli, P', Notterfiede, C. B., Pascale, E. Placentini, F., Pogosyan, D). , Polenta, G., Prunet, S. Ricciarli, S. Romeo, G., Ruhl, J. F., Santini, P. , Tegmark, M. , Veneziani, M. , Vittorio, N. "A Measurement of the CMB EE Spectrum from the 2003 Flight of BOOMERANG". Ap. $647,813-822(2006)$

[5] Page, L., Hinshaw, G., Komatsu, F., Nolta, M. R., Spergel, D. N. , Bennett, C. I., Barnes, C. , Bean. R. Doré, O., Dunkley, J. Halpern, M., Hill, R. S., Jarosik, N., Kogut, A. , Jinon, M. , Meyer, S. S. . Odegard, N., Peiris, II. V. Tucker, G. S., Verde, L., Weil, J. L., Wollack, E., Wright, E. L., "Three-Year Wilkinson Microwave Anisotropy Probe (WMAP) Observations: Polarization Analysis", Ap./S, 170, 335j 376 $(2007)$

[6] Wi. J. H. P., Zuntz, J., Abroc M. E., Ade, P. A. R. , Bock, J., Borrill, J, Collins, J., Hanany, S. . Jaffe. A. H., Johnson, B. R., Jones, T., Lee, A. T., Matsmmm, T., Rabii, B. , Renbarger, T., Richards, P. I. , Smoot, G. F., Stompor, R., Tran, II. T. , Winant, C. D. "MAXIPOL: Data Analysis and Results", Ap.J, $665,55-66(2007)$

[7] Bischoff, C., Hyatt, I., MeNahon, J. J., Nixon, G. W., Sambteben, D), Suith, K. M., Vanderlinde, K. Barkats, D. , Farese, P., Gaier, T. , Gundersen, J. O., Hedman, M. M. , Staggs, S. T. , Winstein, B., "New Measurements of Fine-Scale CNB Polarization Power Spectra from CAPMAP at Both 40 and $90 \mathrm{GH} z ", A p J$. 684, $771-789(2008)$

[8] Pryke, C. , Ade, P. , Bock, J., Bowden, M. , Brown, M. L. , Cahill, G. , Castro, P. G. , Church, S. . Culverhonse, T., Friedman. R. Ganga, K., Gear, W. K. Gupta, S. , Hinderks, J. , Kovac, J. , Lange. A. E., Leitch, E., Melhuish, S. J., Memari, Y., Murphy, J. A. Orl,o, A. , Schwarz, R. , O'Sullivan, C. , Piccirillo, L. , Rajguru, N. , Rushohne, B. , Taylor, A. N., Thompson, K. L., Turner, A. H. , Wu, E. Y. S. , Zemcov, M. "Second and Third Season QUaD Cosmic Microwave Background Temperature and Polarization Power Spectra", ApJ, 692, 1247-1270(2009)

Proc. of SPIE Vol. $7741 \quad 77412 \mathrm{H}-8$ 
[9] Wadefalk, N., Mellberg, A., Angelov, I., Barsky, M., Bui, S., Choumas, E., Grundbacher, E., Lai, R., Rorsman, N., Starski, Pjotr, Stenarson, J., Streit, D., Zirath, H., "Cryogenic, Wideband, Ultra-Low Noise IF-Amplifiers Operating at Ultra-Low DC Power", IEEE Trans. Microw. Theory Tech., 51, 1705-1711, (2003)

[10] Weinreb, S., Lai, R., Erickson, N.. Gaier, T., Wielgus, J., "W-band InP Wideband MMIC LNA with $30 \mathrm{~K}$ Noise Temperature", IEEE MTT-S International, 1, 101-104 (1999)

[11] Deal, W.R., Din, S., Radisic, V., Padilla, J., Mei, X.B., Yoshida, W., Liu, P.H., Uyeda, J.,Barsky. M., Gaier, T., Fung, A., Samoska, L., Lai, R., Demonstration of a sib-millimeter wave integrated circuit (SMMIC) using InP HENT with a 35-nm gate, in Proc. IEEE Compound Semicond. Integr. Circuit. Conf., $3336(2006)$

[12] Bryerton, E., Mei, X., Kim, Y.-M., Deal, W., Yoshida, W., Lange, M., Uyeda, J., Morgan, M., Lai, R., "A W-Band Low-Noise Amplifier with 22K Noise Temperature", IEEE MTT-S International, 681-684 (2009)

[13] Buder, 1., "Q/U Inaging ExperimenT (QU1ET): a gromel-based probe of cosmic microwave backgromnd polarization", Proc. SPIE, $7741(2010)$

[14] Dumoulin, R. N., "Responsivity Calibration of the QUIET Q-band Array", Proc: SPIE, 7741 (2010)

[15] Monsalve, R., "Bean characterization for the QUET Q-Band instrunent using polarized and unpolarized astronomical sources", Proc. Sl'IE, $7741(2010)$

[16] http://quiet.uchicago.edu.

[17] Dicke, R. H., "The Measmrement of Thermal Radiation at Microwave Frequencies," Reviem of Scientific: In.struments 17, 268-275) (July 1946).

[18] Gaier, T. C., "Coherent radioneters for cosmic microwave background polarization detection," in [Polarimetry in Astronomy. Edited by Silvano Fineschi. Procedings of the SPIE, Volume 4843, pp. 296-304 (20093).1. Fineschi, S., al., Presented at the Society of Photo-Optical Instrumentation Engineers (SPIE) Conference? 4843, $296 \cdot 304$ (Fet), 2003).

[19] Barkats, D., CAPMAP': A wew instrument to measure the e-mode CMB polarization on angular scales of 4 feet to 40 feet, PhD thesis. PRINCETON UNIVERSITY (Aug. 2004). 